\title{
Antithrombotic Therapy After Cardiac Surgery: Role of Different Oral Anticoagulants
}

\author{
Vincenzo Caruso ${ }^{1}$, Robert Leatherby ${ }^{2}$, Muhammad Usman Shah $^{3}$, Sudhir Bhusari ${ }^{3}$, Arvind \\ Singh $^{3}$, and Hasnat Khan ${ }^{3}$ \\ ${ }^{1}$ Guy's and Saint Thomas' Hospitals NHS Trust \\ ${ }^{2}$ Wythenshawe Hospital \\ ${ }^{3}$ Basildon and Thurrock University Hospitals NHS Foundation Trust
}

July 2, 2020

\begin{abstract}
Background and aim of the study: Aim of this study is to compare the incidence of postoperative bleeding events, identified as pericardial effusion, for those patients undergoing cardiac surgery and discharged on vitamin K antagonist (VKA) versus those discharged on NOACs. Methods: This was a retrospective observational cohort study; from July 2017 to July 2019 , all the patients who underwent any cardiac surgical procedure and discharged on any oral anticoagulant, were rolled in the study. The study variables and setting followed the STROBE checklist. The final cohort was constituted by 382 patients (mean age $70 \pm 11.2$ years); $260(68.1 \%)$ patients were discharged on VKA and $122(31.9 \%)$ were discharged on NOACs. The primary end point was the incidence of major postoperative bleeding, defined as pericardial effusion requiring surgical re-exploration. The key secondary composite end point was the late re-admission for pericardial effusion. Results: The overall incidence of in-hospital immediate bleeding events, with need of re-exploration for pericardial effusion, was $4.7 \%(\mathrm{n}=18)$. The incidence of re-admission for pericardial effusion was $3.1 \%(n=12)$. Eight of those patients had surgical re-exploration: four patients were discharged on NOACs and the remnant four ones were discharged on VKA. No significant relationships were observed between the different oral anticoagulants and the incidence of pericardial effusion, at any time. No ischemic and thromboembolic events were recorded. Conclusions: The use of non-vitamin K antagonist oral anticoagulant, in post cardiac surgery patients, does not increase the incidence of major bleeding events, intended as immediate or late pericardial effusion.
\end{abstract}

\section{INTRODUCTION}

The novel non-vitamin $\mathrm{K}$ antagonist oral anticoagulant (NOACs) are now widely accepted as valid alternative of vitamin $\mathrm{K}$ antagonist (VKA) in the treatment of non-valvular atrial fibrillation and deep venous thrombosis ${ }^{1-2}$. Recently, subgroup analyses of randomized trial support initial evidence of the use of NOACs in patients with valvular $\mathrm{AF}$, if not associated with degenerative mitral stenosis, or after three months post bio-prosthesis replacement ${ }^{3}$.

Their role after cardiac surgery still represents a challenge choice for the heart team in regards of the risks of bleedings events; objective of this study is to compare the incidence of pericardial effusion in patients started on oral anticoagulant after cardiac surgery, to assess if their use is safe and reproducible.

\section{MATERIALS AND METHODS}

\section{Data source}

From July 2017 to July 2019, data from a single institution were collected in an open, double arm, retroprospective manner. The final database was constituted by pre-operative demographic variable, intra-operative interventional data and post-operative measurements. 
Only the patients who underwent cardiac surgery and discharged with oral anticoagulant were rolled in the study; patients with preexisting bleeding disorders were excluded. Pre-operative use of any oral anticoagulant did not constitute an exclusion criteria. All major, elective, cardiac surgical procedures were considered; patients who underwent urgent or emergency operation were excluded from the study.

The VKA included exclusively warfarin. The NOACs used were dabigatran, rivaroxaban and apixaban.

Reasons for antithrombotic treatment at discharge were atrial fibrillation, deep venous thrombosis, atrial myxoma and left ventricular thrombus.

A bleeding event was defined as evidence of pericardial effusion, with or without need of surgical intervention. The incidence of pleural effusion, cerebral and abdominal hemorrhagic events were recorded but not reported as not primary outcomes of the present study.

All the ischemic or thromboembolic events were collected and reported. As ischemic event it was considered the incidence of cerebrovascular events, bowel ischemia, lower limb ischemia or myocardial infarction. A thromboembolic event was defined as incidence of deep venous thrombosis, pulmonary embolism, cerebral infarct or systemic venous thrombosis.

The primary end point was the incidence of immediate postoperative bleeding, defined as pericardial effusion requiring intra-hospital re-exploration. This term was used to define any pericardial drainage performed before discharge; this was then differentiated as early, if within the first 72 hours from surgery, or late, if after 72 hours.

The key secondary composite end point was the late re-admission for pericardial effusion, with or without need of surgical drainage.

\section{Statistical analysis}

The SPSS ${ }^{\circledR}$ software, version 26.0 (IBM Corporation, Armonk, New York), was used for statistical analysis. Continuous variables are presented as mean \pm standard deviation, and categorical variables are presented as absolute numbers and/or percentages.

A linear regression was used to assess statistically significant between continuous variable, while a chi square test and logistic regression was used for categorical variables. For all tests, a $p$-value $<0.05$ was considered statistically significant.

\section{RESULTS}

The final cohort was constituted by 382 adult patients; of those patients, 260(68.1\%) were discharged on VKA and 122(31.9\%) were discharged on NOACs.

Pre-operative use of oral anticoagulant was recorded in 193 patients (73.2\%) for chronic AF, previous mechanical valve or deep venous thrombosis (Table 1).

In the overall cohort no ischemic or thromboembolic event were recorded both in the immediate postoperative course, both at median follow-up. The median length of stay was 9.5 days (IQR: 7-14). There was no intra-hospital mortality and all the patients were alive at the follow-up (median 23.9 months, IQR:18-30).

\section{Covariates analysis:}

The main reason for anticoagulation, in the overall cohort, was $\mathrm{AF}(\mathrm{n}=308,80.6 \%)$.

In the VKA's group, warfarin was started for AF in 192 patients(73\%); 111(57\%) of those patients had chronic $\mathrm{AF}$ and they were on anticoagulation prior to surgery, either with Warfarin $(\mathrm{n}=98,89 \%)$ or NOACs$(\mathrm{n}=13$, $11 \%)$.

In the NOACs'cohort, anticoagulation was required in 116 patients (95\%) for AF. NOACs was used de novo in 49 patients (42\%), while sixty-two of those patients (57\%) were on NOACs prior to surgery. 
All the patients with a mechanical valve were started on Warfarin at postoperative day two ( $\mathrm{n}=62,23 \%)$.

Warfarin was used, with a limited three months length, in six patients $(4 \%)$ with left ventricular thrombus and atrial myxoma.

\section{Intra-hospital re-exploration}

The overall rate of intra-hospital re-exploration, for all causes - pericardial effusion, delayed sternal closure, graft failure or cardiac arrest - was $6.5 \%(\mathrm{n}=25)$.

In this cohort, the incidence of pericardial effusion, was $72 \%(\mathrm{n}=18)$. Ten patients $(55 \%)$ returned in theatre within the first 72 hours from surgery. In these cases, no significant relationships were observed between the incidence of re-exploration and pre-operative use of anticoagulants ( $p=.150$; Odd Ratio: 0.52(0.2-1.2); NOACs: $\mathrm{n}=2,1.9 \%$, ; Warfarin: $\mathrm{n}=1,0.9 \%$ ).

Nine patients required re-exploration after 72 hours (mean post-operative day of 11.8 \pm 4.3 ). Warfarin was already started for seven patients with a median value of INR of 1.8 (IQR: 1.2-2.6). Higher the INR, bigger was the effusion drained (median:800mls, IQR: 400-1250), but this was not statistically significant $(r=0.37$, $\left.\mathrm{R}^{2}=0.13 p=0.368, \mathrm{n}=9\right)$.

Major aortic surgery was statistically related to a higher incidence of re-exploration when compared with the other surgical procedures ( $p=.011$; Odd Ratio: 3.4(1.3-8.4), (Table 2).

\section{Re-admission}

The incidence of re-admission for pericardial effusion was $3.1 \%(\mathrm{n}=12)$ at a median of 11 days(IQR: 7.5-99). All the patients expect four ( $\mathrm{NOACs}=3,27 \%$; Warfarin=1,9\%), underwent surgical drainage. Four of those patients were discharged on NOACs (Apixaban=2, 25\%, Rivaroxaban=2, 25\%).

In the NOACs group the median effusion drained was 650mls (IQR: 525-775). In the Warfarin group, the median INR was 2.2 (IQR: 1.7-3.4). Also, in this case, a higher INR was associated with a bigger effusion (median:800mls, IQR: 750-1100), but this was not statistically significant $\left(r=0.8, \mathrm{R}^{2}=0.65 p=.192, \mathrm{n}=4\right.$ ).

\section{CONCLUSION}

Different studies have showed the safety of NOACs as alternative to warfarin in the treatment of AF and deep venous thrombosis ${ }^{1-3}$. The updated guidelines on the management of atrial fibrillation now recommends the new oral anticoagulants (NOACs) as the preferred anticoagulant to warfarin, unless patients have moderate to severe mitral stenosis or they have an artificial heart valve ${ }^{4}$. Their use after cardiac surgery seems to be safe as showed by a retrospective study that evaluated the length of stay, the post-operative bleeding and the cost of the therapy ${ }^{5}$.

This retrospective study evaluates the incidence of post-operative pericardial effusion as a measure of bleeding event. No statistical differences were observed in the two cohorts examined, with an incidence similar between NOACs and Warfarin; despite the pre-operative use of NOACs seems to increase the incidence of early reexploration, this was not statistically significant. A strong association was instead noted between pericardial effusion and type of surgery. More interestingly, it was observed that a high value of INR correlated with the effusion drained; plus, almost all the patients re-admitted and on Warfarin, required a surgical re-exploration.

In term of re-admission, after initial discharge, this was similar between NOACs and warfarin, with no significant differences.

At the end, from this retrospective study, appears that the use of NOACs in post cardiac surgery, appeared to be a safe and valid alternative to warfarin, in term of incidence of pericardial effusion and need of surgical drainage.

In patients undergoing surgery for valvular disease, expect in those with mechanical valve, the use of NOACs appears safe as immediate treatment. 
This study has some limits: the overall cohort is small with a prevalent warfarin group; second, the incidence of thromboembolic events and/or other hemorrhagic events, was not considered as primary outcome.

Despite this, at follow-up, none of the patients in the study showed significant complications, redo-surgery for graft or valve failure and/or mortality.

\section{ACKNOWLEDGEMENTS}

No acknowledgements

\section{CONFLICT OF INTEREST}

None

\section{AUTHOR CONTRIBUTION:}

Dr Caruso: partecipate in concept and design, analysis and interpretation, writing the article, critical revision of the article, final approval of the article, data collection, statistical expertise, literature search.

Dr Leatherby: partecipate in concept and design, analysis and interpretation, writing the article, critical revision of the article, final approval of the article, data collection.

Dr Shah: partecipate in concept and design, analysis and interpretation, writing the article, critical revision of the article, final approval of the article, data collection.

Mr Bhusari: partecipate in concept and design, analysis and interpretation, critical revision of the article, final approval of the article.

Mr Singh: partecipate in concept and design, analysis and interpretation, critical revision of the article, final approval of the article.

Mr Khan: partecipate in concept and design, analysis and interpretation, critical revision of the article, final approval of the article.

\section{REFERENCES}

1. Patel MR, Mahaffey KW, Garg J, Pan G, Singer DE, Hacke W, et al.. The ROCKET AF Steering Committee . Rivaroxaban versus warfarin in nonvalvular atrial fibrillation. N Engl J Med 2011; 365: 883-891

2. Schulman S, Kearon C, Kakkar AK, Mismetti P, Schellong S, Eriksson H, et al. Dabigatran versus warfarin in the treatment of acute venous thromboembolism. N Engl J Med 2009; 361: 2342-2352.

3. Avezum A, Lopes RD, Schulte PJ, Lanas F, Gersh BJ, Hanna M, et al. Apixaban in comparison with warfarin in patients with atrial fibrillation and Valvular heart disease: findings from the Apixaban for reduction in stroke and other Thromboembolic events in atrial fibrillation (ARISTOTLE) trial. Circulation. 2015;132(8):624-32

4. Craig T. January, et al. 2019 AHA/ACC/HRS Focused Update of the 2014 AHA/ACC/HRS Guideline for the Management of Patients with Atrial Fibrillation: A Report of the American College of Cardiology/American Heart Association Task Force on Clinical Practice Guidelines and the Heart Rhythm Society in Collaboration with the Society of Thoracic Surgeons. Circulation. 2019;140:e125-e151

5. Anderson E., Johnke K., Leedhal D., Glogoza M., Newman R., Dyke C. Novel oral anticoagulants vs warfarin for the management of postoperative atrial fibrillation: clinical outcomes and cost analysis. J. Am Surg. 2015; 210(6):1095-1103.

\section{Table Legend:}

TABLE 1: Baseline characteristics, preoperative medications and cardiac surgical procedure.

TABLE 2: Univariate analysis of risk factors: Surgery and re-exploration for pericardial effusion. 
TABLE 1: Baseline characteristics, preoperative medications and cardiac surgical procedure.

\begin{tabular}{lll}
\hline covariates & vka $(\mathrm{N}=260)$ & noac $(\mathrm{N}=122)$ \\
\hline Age (years) & $68.1 \pm 12.1$ & $74.1 \pm 7.9$ \\
Gender (\%) & $87(33.4)$ & $32(26.2)$ \\
Preoperative Anticoagulant (\%) & $120(46.1)$ & $73(59.8))$ \\
Warfarin (\%) & $98(81)$ & $4(5)$ \\
NOAC (\%) & $22(19)$ & $69(95)$ \\
Anti-platelets (\%) & $84(32)$ & $41(33)$ \\
CABG alone & 92 & 41 \\
AVR/AVR+CABG Tissue AVR & $139(96 / 43) 8851$ & $49(31 / 18) 490$ \\
Mechanical AVR & & $43(34 / 9) 3490$ \\
MVR/MVR+CABG MV & $80(70 / 10) 462410$ & \\
Repair Tissue MVR Mechanical & & $4($ Repair $)$ \\
MVR & $3($ Repair $)$ & 3 \\
MVR +/- TVR & 48 & 4 \\
Major Aortic Surgery & 9 & 3 \\
Other Surgery & 14 & \\
Redo & &
\end{tabular}

Legends: $C A B G=$ Coronary artery by-pass graft; $A V R=$ Aortic valve replacement; $M V R=$ Mitral valve repair/replacement; $T V R=$ Tricuspid valve repair/replacement.

* Other Surgery= Ventricular septal defect closure, Pericardiectomy, Morrow's procedure.

TABLE 2: Univariate analysis of risk factors: Surgery and re-exploration for pericardial effusion.

\begin{tabular}{lll}
\hline covariates & $p$ value & Odd ratio(95\% CI) \\
\hline CABG alone & .527 & $0.7(0.3-1.6)$ \\
AVR/AVR+CABG & .150 & $1.9(0.8-4.4)$ \\
MVR/MVR+CABG & .825 & $0.8(0.3-1.9)$ \\
MVR +/- TVR & .112 & $2.3(0.8-4.4)$ \\
Major Aortic Surgery & .011 & $3.4(1.3-8.4)$ \\
\hline
\end{tabular}

Legends: $C A B G=$ Coronary artery by-pass graft; $A V R=$ Aortic valve replacement; $M V R=$ Mitral valve repair/replacement; $T V R=$ Tricuspid valve repair/replacement.

\section{Hosted file}

TABLE 1.docx available at https://authorea.com/users/325508/articles/465457-antithrombotictherapy-after-cardiac-surgery-role-of-different-oral-anticoagulants

\section{Hosted file}

TABLE 2.docx available at https://authorea.com/users/325508/articles/465457-antithrombotictherapy-after-cardiac-surgery-role-of-different-oral-anticoagulants 